\title{
Giant Brillouin Amplification in Gas Using Hollow-core Fiber
}

\author{
Luc Thévenaz, Fan Yang, Flavien Gyger \\ Ecole Polytechnique Fédérale de Lausanne, Group for Fibre Optics, EPFL SCI-STI-LT, Station 11, 1015 Lausanne, Switzerland \\ Author e-mail address: Luc.Thevenaz@EPFL.CH
}

\begin{abstract}
Optical amplification in hollow core fibers has been achieved using stimulated Brillouin scattering directly in the gaseous medium. More than $50 \mathrm{~dB}$ optical gain is observed over $50 \mathrm{~m}$ of fiber using $200 \mathrm{~mW}$ of pump power. (c) 2021 The Author(s)
\end{abstract}

\section{Preamble}

Optical amplification using gas as amplifying medium is something known for a long time: every person educated in optics is most probably familiar with He-Ne lasers and their omnipresent tubes have been the favorite light source in optical labs until the advent of visible semiconductor lasers. The amplification in such a gas medium is performed very classically by creating a population inversion between atomic or molecular energy levels. Here we shall show that gases can also amplify light by using an entirely different process, based on the power transfer between an intense pump beam and a distinct signal wave to be amplified, using a nonlinear coupling.

This approach turns out to be very attractive on many aspects: the linear gains achieved can significantly outperform those obtained using atomic transitions. The amplification is moreover not limited to photons with wavelength matching the energy gap between atomic or molecular transitions, so that the gain process is totally achromatic within the transparency window of the gas - normally extremely wide - and is only fixed by the pump wavelength.

The principle underlying such a nonlinear coupling relies primarily on the simple effect observed in a "heat haze", in which the light can be significantly deviated by fluctuations of the gas density. The gas can be compressed in a controlled and ordered manner by exploiting the particle dragging effect using optical forces generated in a gradient of light intensity, like in optical tweezers. This periodic compression generates a refractive index grating realizing the coupling between pump and signal via a Bragg reflection. The amplitude of this grating can be massively and resonantly enhanced by moving the light intensity pattern at the acoustic velocity in the gas medium: this is the well-known process of stimulated Brillouin scattering

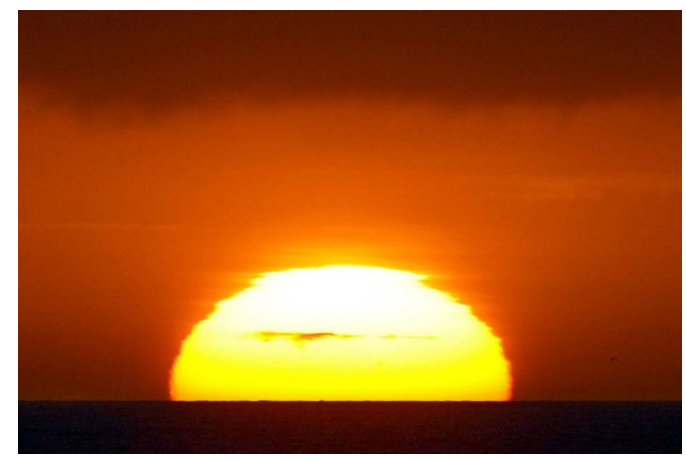
(SBS) that has proved to show the largest efficiency among all nonlinear optical effects.

The advantages of coherent nonlinear amplification are numerous: gain can be induced in the medium at virtually any wavelength, amplification is distributed, spectrally-selective, directional and, in the case of SBS, the gain spectral profile can be precisely customized by a proper pump modulation. More importantly, this offers an elegant and efficient solution for the intractable problem of direct optical amplification in hollow core fibers, in which an unprecedented gain in the gas trapped in the core has been demonstrated using SBS [1].

Backward stimulated Brillouin scattering (SBS) in gases is a very weak effect under ambient pressure, but turns out to show very high interaction strengths under high gas pressure conditions and linear gains up to 1000X larger than in silica can be realistically achieved, as shown in Fig. 1. This value is still 10 times larger than in chalcogenide glasses, considered like the most efficient waveguide materials for SBS interactions [2]. This simply outperforms the best achievements using any other nonlinear interactions in the best materials for optical waveguides.

Stimulated Brillouin scattering cannot be reduced to a mere amplification process, since it has demonstrated its potentialities to realize advanced functions [3]. This novel gas-based Brillouin platform can be the foundation of many potential applications: amplifiers, highly coherent Brillouin gas lasers, slow \& fast light, microwave filters, tunable delay lines, light storage, all-optical calculus and of course sensing [4]. The same functions as those realized 


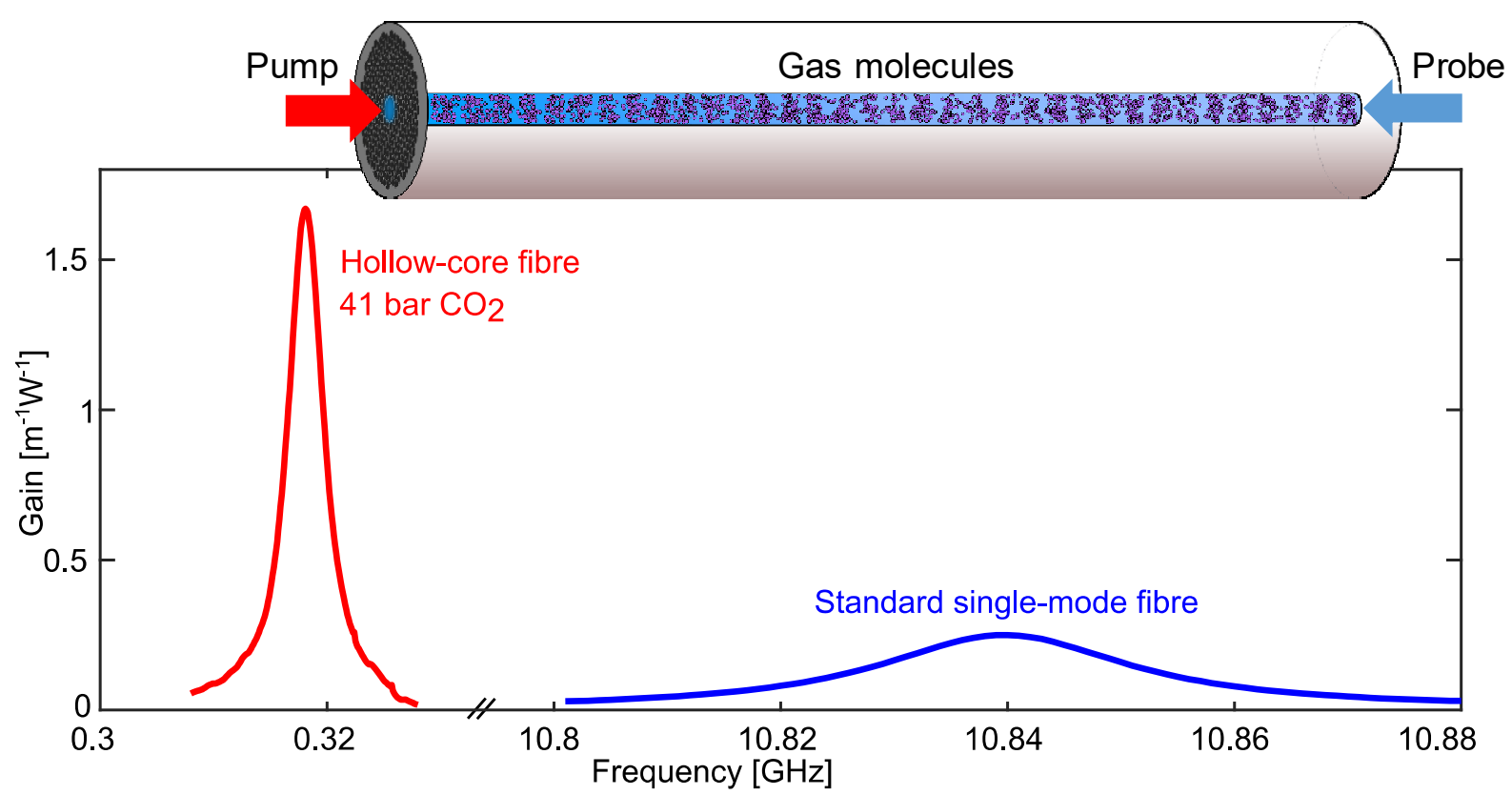

Fig. 1. Gain measured in a hollow-core photonic bandgap fiber (red) filled with $\mathrm{CO} 2$ gas at high pressure, as function of the optical frequency detuning between the pump and the counterpropaging amplified probe signal. For comparison the gain measured in a solid core silica fiber is also represented (blue). It demonstrates that a substantially higher gain is achieved in the gas, showing a narrower gain linewidth obtained at a smaller frequency detuning. The horizontal scale is broken for a better visualization, but kept unmodified.

in solid core silica fibers can therefore be implemented in hollow-core fibers, with all inherent assets of fiber-based optics and the key advantage to realize the same response with a product pump power $\mathrm{x}$ fibre length $1000 \mathrm{X}$ smaller.

\section{Principle \& Realization}

The issue of optical amplification in hollow-core fibers has already given rise to sustained efforts and interesting results have been reported, mostly using atomic transitions in a low pressure gas (like in classical gas lasers) [5] or Raman gain in hydrogen [6,7]. The obtained gains remain modest in both cases when compared to solid-core solutions, with the specific penalties of amplification at fixed wavelengths for atomic transitions and the issue of fast hydrogen permeation through the glass walls for Raman amplification.

The strength of the Stimulated Brillouin interaction is directly related to the absolute change of refractive index per unit power of the interacting waves. It is essentially proportional to the molecular polarizability, the nominal density of the medium and its compressibility [8]. There are specific differences between solid and gaseous media: in a solid material, the density is high, but the compressibility is low. The reverse situation is observed in gases. So nominally, there is no fundamental reason to make the Brillouin gain vanishingly small in gases.

Actually, at ambient conditions, the gain in compressibility does not compensate the loss in density and the Brillouin interaction strength is effectively some 2 orders of magnitude smaller than in a solid. However, rising the pressure increases the density proportionally, but affects marginally the compressibility, so the interaction strength is expected to grow proportionally to pressure.

The situation is in reality even more favorable, since the acoustic loss decreases proportionally to the pressure. The pressure thus contributes a second time to increase the acoustic amplitude and the strength of the interaction eventually depends quadratically on the pressure. Concretely, by rising the pressure to a few tens of bars, Brillouin gains comparable to solid materials can be obtained. They may even be largely overcome by further rising the pressure to hundreds of bars, which is proved perfectly sustainable in hollow-core fibers [9].

These intuitive predictions have been fully confirmed experimentally and, by filling the hollow-core fiber with $\mathrm{CO}_{2}$ at a pressure of 41 bar, a very important net amplification up to $53 \mathrm{~dB}$ could be obtained over $50 \mathrm{~m}$ of fiber with moderate pump powers, as shown in Fig. 2. 

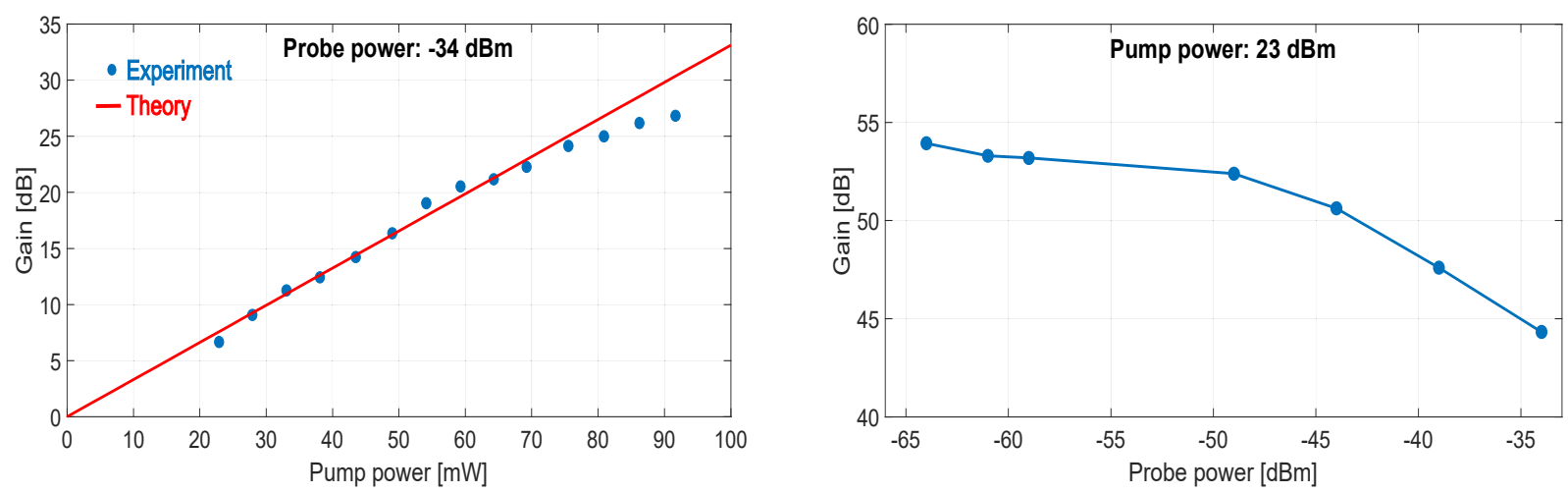

Fig. 2 Net optical gain on a weak probe signal obtained by SBS along $50 \mathrm{~m}$ of hollow core photonic bandgap fiber filled by $\mathrm{CO}_{2}$ at a pressure of 41 bar. Left: constant probe power and varying pump power. The red line is not a fit, but is obtained from the theoretical prediction. Right: varying probe power and constant pump power, showing gain saturation through pump depletion for a probe power in the nanowatt range.

These results lead to formulate the following comments and highlight the specificities of SBS amplification in gases:

- The frequency difference between the 2 interfering lightwaves, corresponding to the frequency of the acoustic wave and called Brillouin shift, is tightly related to the acoustic velocity, in direct proportion. It is high in a solid material, giving a Brillouin shift of some $10 \mathrm{GHz}$ in silica, but the sounds propagate much more slowly in a gas, with corresponding Brillouin shifts in the range $0.1-1 \mathrm{GHz}$. This lower frequency may facilitate the synthesis of the different optical signals without using microwave modulation, but makes the spectral separation of these signals in the optical domain much more challenging.

- The Brillouin shift is specific to a given gas and can be finely customized over a broad spectral range by a proper gas mixture. The density also depends on the gas species and this will modify the SBS response, accordingly. For an ideal gas, the density is proportional to the molecular mass $M$, so larger gains can potentially be obtained by using heavy molecules, with again a squared dependence on $M$. It must be noted that gases with large molecules normally show a liquefaction pressure that is relatively low, cancelling most benefits from the higher molecular mass by setting a maximum value for the pressure and thus the gain. A lighter molecule showing no liquid phase at ambient temperature may eventually deliver higher gains by simply rising the pressure. More complex molecules are also more likely to present spectral absorption lines near the wavelength of interest, which may induce a spurious attenuation over a wide spectrum as a result of the large pressure line broadening. Gain can also be scaled by varying pressure and mixture.

- Other nonlinearities and optical damage are virtually inexistent in gases, giving space for higher powers.

The envisioned potentialities are very large and the implementations practically realistic, with huge amplification in the range of $50 \mathrm{~dB}$ over less than $100 \mathrm{~m}$ of fibre and sub-watt pumping powers.

This work is supported by the Swiss National Science Foundation under grant agreement No. 178895.

\section{References}

[1] F. Yang, F. Gyger, and L. Thévenaz, "Intense Brillouin amplification in gas using hollow-core waveguides," Nat. Photonics 14, 700-708 (2020).

[2] K. S. Abedin, "Observation of strong stimulated Brillouin scattering in single-mode As2Se3 chalcogenide fiber," Opt. Express 13, 10266$10271(2005)$.

[3] M. Santagiustina, S. Chin, N. Primerov, L. Ursini, and L. Thévenaz, "All-optical signal processing using dynamic Brillouin gratings," Sci. Rep. 3, 1594 (2013).

[4] B. J. Eggleton, C. G. Poulton, P. T. Rakich, M. J. Steel, and G. Bahl, "Brillouin integrated photonics," Nat. Photonics 13, 1-14 (2019).

[5] M. R. A. Hassan, F. Yu, W. J. Wadsworth, and J. C. Knight, "Cavity-based mid-IR fiber gas laser pumped by a diode laser," Optica 3, 218221 (2016)

[6 F. Benabid, J. C. Knight, G. Antonopoulos, and P. S. J. Russell, "Stimulated Raman Scattering in Hydrogen-Filled Hollow-Core Photonic Crystal Fiber," Science 298, 399-402 (2002).

[7] M. K. Mridha, D. Novoa, P. Hosseini, and P. S. J. Russell, "Thresholdless deep and vacuum ultraviolet Raman frequency conversion in hydrogen-filled photonic crystal fiber," Optica 6, 731-734 (2019).

[7] W. H. Renninger, R. O. Behunin, and P. T. Rakich, “Guided-wave Brillouin scattering in air,” Optica 3, 1316-1319 (2016).

[8] E. E. Hagenlocker and W. G. Rado, "Stimulated Brillouin and Raman scattering in gases," Appl. Phys. Lett. 7, 236-238 (1965).

[9] P. S. J. Russell, P. Hölzer, W. Chang, A. Abdolvand, and J. C. Travers, "Hollow-core photonic crystal fibres for gas-based nonlinear optics," Nat. Photonics 8, 278-286 (2014). 